\title{
Straight and crosier-shaped catheter techniques for phrenic nerve stimulation during cryoballoon pulmonary vein isolation for the treatment of atrial fibrillation
}

\author{
Krzysztof Kaczmarek1', Bartosz Żuchowski², Iwona Cygankiewicz'1, Anna Krakowiak1, \\ Jerzy Krzysztof Wranicz ${ }^{1}$, Andrzej Wykrętowicz², Paweł Ptaszyński ${ }^{1}$ \\ 1 Department of Electrocardiology, Medical University of Lodz, tódź, Poland \\ 2 Department of Cardiology-Intensive Therapy, Poznan University of Medical Sciences, Poznań, Poland
}

\section{KEY WORDS}

atrial fibrillation, catheter ablation, catheter position, cryoballoon, phrenic nerve stimulation
Correspondence to: Bartosz Żuchowski, MD, Department of Cardiology-Intensive Therapy, Heliodor Święcicki University Hospital, ul. Przybyszewskiego 49, 60-355 Poznań, Poland, phone: +48618691391, email: b.zuchowski@gmail.com Received: May 6, 2019. Revision accepted: July 22, 2019 Published online: July 22, 2019. Kardiol Pol. 2019; 77 (9): 868-874 doi:10.33963/KP.14908 Copyright by the Author(s), 2019

\begin{abstract}
BACKGROUND Cryoablation is an effective and safe method of pulmonary vein isolation (PVI) for the treatment of atrial fibrillation (AF). However, monitoring of phrenic nerve function during cryoballoon PVI remains an important issue.

AIMS We aimed to compare 2 techniques of phrenic nerve stimulation (PNS) with the use of either a straight or a crosier-shaped decapolar deflectable catheter.

METHODS The study included 218 patients (mean [SD] age, 61.8 [10.9] years; 87 women) referred for cryoballoon PVI for AF. Patients were randomly assigned to PNS with either a straight or a crosier-shaped catheter.

RESULTS The groups did not differ in demographic characteristics or PVI effectiveness. The current threshold during PNS was lower in the group in which the crosier-shaped catheter was applied (mean [SD], 6.7 [4.9] mA vs 4.8 [3.7] $\mathrm{mA} ; P<0.01$ ). In this group, the procedure time was shorter (mean [SD], 72.6 [22.8] min vs $64.4[14.8] \mathrm{min} ; P<0.01)$, fewer repositioning maneuvers were required ( $31.8 \%$ vs $19.4 \% ; P<0.05$ ), and atrial capture during PNS was observed more frequently (11.5\% vs $29.6 \% ; P<0.01)$.

CONCLUSIONS Straight and crosier-shaped catheter techniques are equally effective in monitoring for phrenic nerve palsy. The crosier-shaped catheter maintains its position better, thus leading to fewer dislocations and requiring fewer correction maneuvers, which might correlate with shorter procedure time. Moreover, this technique provides lower pacing thresholds. Both techniques may be safely used in patients with AF, and if stable phrenic nerve capture cannot be achieved, switching to another technique seems reasonable.
\end{abstract}

INTRODUCTION Pulmonary vein isolation (PVI) has become a standard treatment approach for symptomatic, drug-refractory paroxysmal atrial fibrillation (AF). The experts recommend catheter ablation for paroxysmal (class I; level of evidence: A) and persistent (class IIa; level of evidence: B) AF. Catheter ablation should be also considered prior to initiation of antiarrhythmic treatment in symptomatic paroxysmal or persistent AF (class IIa; levels of evidence: $\mathrm{B} / \mathrm{C}) .{ }^{1}$ Cryoablation of pulmonary veins with the use of a balloon catheter is the most popular single-shot ablation technique in AF treatment. In the large FIRE AND ICE trial, ${ }^{2}$ cryoablation was proved to be noninferior to radiofrequency ablation with respect to its efficacy. Moreover, the total procedure time and catheter dwell time in the left atrium were significantly shorter in the cryoballoon group at the cost of longer fluoroscopy time. There was also a significant difference in the occurrence of phrenic nerve injury (PNI). This complication was highly 


\section{WHAT'S NEW?}

We compared 2 catheter techniques for phrenic nerve stimulation (PNS) during cryoballoon pulmonary vein isolation (PVI) for the treatment of atrial fibrillation (AF). We found that with the use of a crosier-shaped catheter, the catheter position was better maintained, fewer correction maneuvers were required, lower pacing thresholds were provided, and atrial capture during pacing was observed more often than with the use of a straight catheter. Moreover, the crosier-shaped catheter technique was correlated with shorter procedure time, which may be due to fewer catheter dislocations. In conclusion, both techniques may be safely used for PNS during cryoballoon PVI in patients with $A F$, and in the case of problems with reaching the stable capture of the phrenic nerve, switching to the other technique seems reasonable.

uncommon in radiofrequency ablation $(0 \mathrm{pa}-$ tients in the FIRE AND ICE trial), while after cryoballoon ablation, phrenic nerve function was impaired in $2.7 \%$ of patients at hospital discharge. As indicated in the literature, the percentage of patients with PNI after cryoablation of the pulmonary veins varies from $2.7 \%$ to $19 \% .^{2-5}$ As reported by Straube et $a l,{ }^{5}$ the phrenic nerve function returned to normal in half of the cases even before hospital discharge, while the remaining instances of palsy usually healed in the first months after the procedure, and a persistent injury was very rare. Phrenic nerve injury that does not resolve within 12 months, and hence may be treated as a permanent injury, occurs in about $0.3 \%$ to $0.4 \%$ of cases. ${ }^{1,2,6}$

Phrenic nerve stimulation (PNS) with simultaneous monitoring of the phrenic nerve function is a well-established technique that leads to a reduction of PNI during cryoablation of the right pulmonary veins. Methods to monitor the function of the phrenic nerve include detecting diaphragmatic contractions by palpation, visualizing diaphragmatic motion by diaphragm fluoroscopy, electromyography with the use of modified precordial electrocardiogram leads (to record the value of the compound muscle action potential [CMAP]), 7,8 auditory cardiotocography, intracardiac echocardiography for visualizing diaphragmatic contractions, and observing the alterations of the waveform in the femoral vein..$^{9-11}$ Because of its simplicity and no need to apply additional devices, palpation is the most common method for monitoring phrenic nerve function; however, assessment of CMAPs has been proved to be more effective in the prevention of PNI.12

For the purpose of PNS, a catheter is usually placed in the superior vena cava above the level of a cryoballoon ${ }^{10,11}$ or in the right subclavian vein..$^{13}$ The optimal site to apply pacing should enable the constant capture of the phrenic nerve with a reasonable pacing threshold and stable catheter position. The procedure is usually conducted with the use of a decapolar deflectable catheter thanks to its versatility in terms of positioning in the caval vein and multiple pacing electrodes at different levels of the vessel.
The deflectable catheter may be placed in the superior vena cava either in a straight position (with distal electrodes oriented cranially) or in a retroflexed, curved position (with distal electrodes oriented caudally), thus assuming the shape of a crosier. The crosier-shaped catheter has a larger area of contact with the vein walls, which may improve its stability. To our best knowledge, no studies comparing these 2 catheter techniques for PNS have been published so far.

METHODS A prospective, dual-center, open-label, experimental study was performed to evaluate differences between applying the straight and crosier-shaped catheters for PNS during cryoballoon pulmonary vein isolation (PVI). The study complied with the Declaration of Helsinki and was approved by the local bioethical committee of the Medical University of Lodz, Poland. All participants gave their informed consent.

The study enrolled consecutive patients scheduled for PVI with cryoablation due to symptomatic, paroxysmal, or persistent $\mathrm{AF}$. As all patients who undergo cryoballoon ablation in our centers routinely undergo PNS, the only exclusion criterion was lack of informed consent. Patients were assigned to the study and control groups in a 1:1 ratio. The primary endpoint was defined as the need to perform any correction maneuvers to maintain phrenic nerve capture. The maneuvers used to continue PNS were determined as follows: 1) correction of the catheter position without terminating cryoballoon application; 2) correction of the catheter position with the concomitant termination of cryoballoon application; and 3) correction of the catheter position that included switching to another pacing technique.

Additionally, we analyzed the following parameters: the threshold of phrenic nerve capture, the time required to position the PNS catheter, the occurrence of phrenic nerve palsy, and the procedure time. The time required to position the catheter was defined as the period needed to achieve the catheter position that provided phrenic nerve capture with an output of $20 \mathrm{~mA}$ and impulse duration of $2 \mathrm{~ms}$. This parameter was then dichotomized as lasting below or above 30 seconds. Following our routine approach, the phrenic nerve was paced at $60 \mathrm{bpm}$ with an output of $20 \mathrm{~mA}$ and impulse duration of $2 \mathrm{~ms}$, independently of the threshold value of phrenic nerve capture.

All procedures were performed by the same operator to limit the occurrence of operator-dependent errors. The main goal of the procedure was the electrical isolation of the pulmonary veins. For this purpose, following our standard of care, a cryoballoon catheter (Arctic Front Advance Cryoballoon Catheter, Medtronic, Minneapolis, Minnesota, United States) was positioned 



FIGURE 1 Phrenic nerve stimulation with a catheter in the straight position: A - anterior-posterior projection, B - left anterior oblique projection, C - right anterior oblique projection
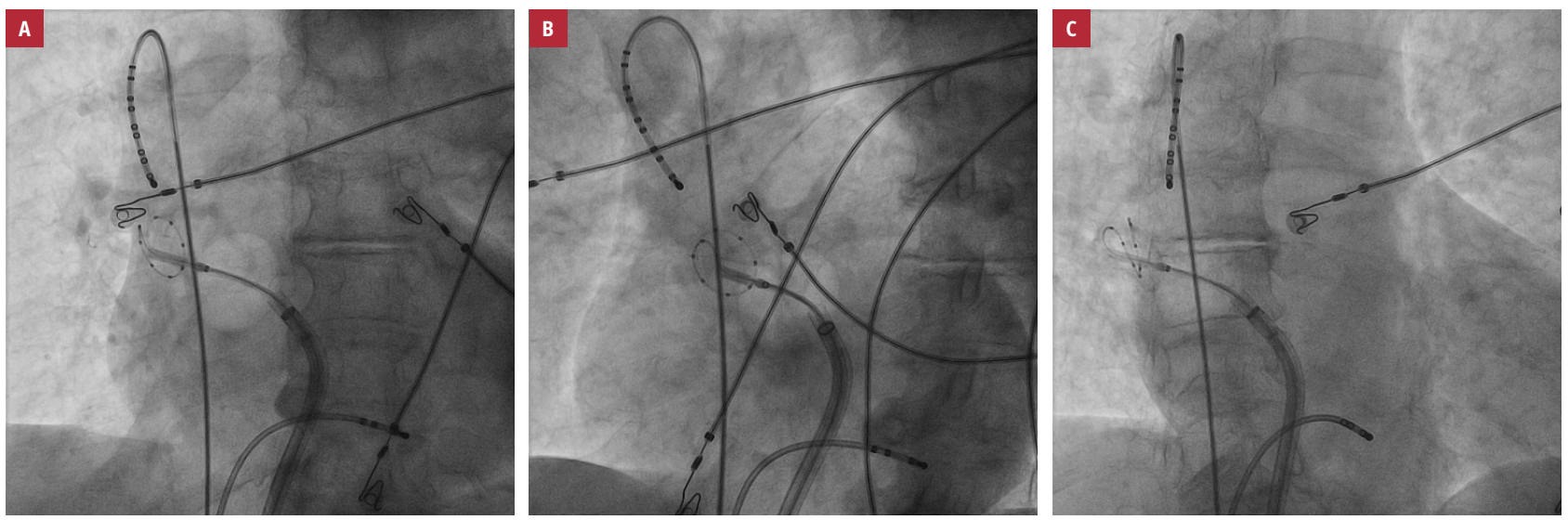

FIGURE 2 Phrenic nerve stimulation with a catheter in the crosier-shaped position: A - anterior-posterior projection, B - left anterior oblique projection, C - right anterior oblique projection

in the ostium of each pulmonary vein and cryoballoon applications were delivered. Electrical isolation was confirmed with a circular mapping catheter (Achieve Mapping Catheter, Medtronic, Minneapolis, Minnesota, United States). The optimal initial positioning of the cryoballoon catheter in the ostium of the pulmonary vein, the repositioning or particular maneuvers of the catheter, as well as an additional cryoballoon application in case of lack of or late PVI were at the discretion of the operating physician. All patients were under conscious sedation. In all cases, during cryoballoon applications in the ostia of the right pulmonary veins, a steerable decapolar PNS catheter was advanced through either the right or left femoral vein to the right atrium. Three different catheters, yet all with the spacing of $5 \mathrm{~mm}$ between pacing poles, were used: Dynamic XT (Boston Scientific, Marlborough, Massachusetts, United States) in 108 patients (49.5\%), Coronary Sinus Decapolar (Hagmed, Rawa Mazowiecka, Poland) in 61 (27.9\%), and Triguy Decapolar (APT Medical, Shenzhen, China) in 49 (22.4\%). During the transseptal puncture and isolation of the left pulmonary veins, the catheter was positioned in the coronary sinus, and prior to the cryoablation of the right pulmonary veins, the device was relocated to the superior vena cava. In the group of patients in which the straight catheter was applied, the catheter was advanced until its proximal pacing electrodes reached the level of the right atrium and the junction of the superior vena cava, and it was minimally bent to get in contact with the vein wall (FGGURE 1). Then, rotation and minor vertical movements of the catheter were performed to find the optimal pacing site around the anterolateral wall of the vein. In the group in which the crosier-shaped catheter was used, the catheter was fully bent in the right atrium and was advanced into the superior vena cava until distal pacing electrodes reached the site located about $1 \mathrm{~cm}$ over the level of the atriovenous junction. Afterwards, the curvature was released so that multiple poles could contact the anterolateral wall of the vein (FGURE2). The optimal pacing site was searched for by rotation and switching of pacing poles. In both groups, the pacing was bipolar and applied from adjacent poles. If a phrenic nerve palsy was observed, cryoballoon application was stopped by pressing a stop button twice. In such a case, the decision as to whether to continue or abandon further cryoballoon application in the ostium of the vein was left at the physician's discretion. 
TABLE 1 Baseline characteristics of study groups by catheter technique used for phrenic nerve stimulation

\begin{tabular}{|c|c|c|c|}
\hline \multicolumn{2}{|l|}{ Parameter } & $\begin{array}{l}\text { Straight catheter } \\
(n=110)\end{array}$ & $\begin{array}{l}\text { Crosier-shaped } \\
\text { catheter }(n=108)\end{array}$ \\
\hline \multicolumn{2}{|l|}{ Age, $y$, mean (SD) } & $61.5(13.6)$ & $62.2(7.2)$ \\
\hline \multicolumn{2}{|c|}{ Body mass index, $\mathrm{kg} / \mathrm{m}^{2}$, mean (SD) } & $29.0(4.3)$ & $29.8(4.6)$ \\
\hline \multirow[t]{2}{*}{ Atrial fibrillation, \% } & Paroxysmal & 79 & 75 \\
\hline & Persistent & 21 & 25 \\
\hline \multicolumn{2}{|c|}{ EHRA score, mean (SD) } & $2.7(0.5)$ & $2.6(0.6)$ \\
\hline \multicolumn{2}{|c|}{$\begin{array}{l}\text { Left ventricular ejection fraction, \%, } \\
\text { mean (SD) }\end{array}$} & $54.8(8.4)$ & $57.4(7.3)$ \\
\hline \multicolumn{2}{|c|}{ Coronary artery disease, n (\%) } & $34(30.9)$ & $30(27.8)$ \\
\hline \multicolumn{2}{|c|}{ Chronic heart failure, n (\%) } & $6(5.5)$ & $6(5.5)$ \\
\hline \multicolumn{2}{|l|}{ Dyslipidemia, n (\%) } & $51(46)$ & $45(42)$ \\
\hline \multicolumn{2}{|l|}{ Hypertension, n (\%) } & $71(64)$ & $69(63.8)$ \\
\hline \multicolumn{2}{|c|}{ Type 2 diabetes mellitus, $\mathrm{n}(\%)$} & $9(8.2)$ & $14(12.9)$ \\
\hline
\end{tabular}

Abbreviation: EHRA, European Heart Rhythm Association

Statistical analysis The statistical analysis was performed using the STATISTICA software, version 12 (StatSoft Inc., Tulsa, Oklahoma, United States). Continuous variables were presented as mean (SD). The $t$ test and Wilcoxon-MannWhitney test were used for a between-group comparison according to data distribution.
Categorical variables were demonstrated as frequency. The $\mathrm{X}^{2}$ test and its modification were used to compare categorical data. The $P$ values of less than 0.05 were considered significant.

RESULTS The study population included 218 consecutive patients ( 87 women) at a mean (SD) age of 61.8 (10.9) years. Patients were assigned to 2 groups in a 1:1 ratio, based on whether a straight or a crosier-shaped catheter was used in PVI. The detailed characteristics of the study groups are shown in TABLE1. The groups did not differ significantly in terms of age, cardiac function, and comorbidities.

During the procedures, $98.8 \%$ of the pulmonary veins were successfully isolated, which required approximately 1.5 cryoballoon applications per vein (mean [SD], 1.4 [0.4]), with the time of a single freeze of approximately $243 \mathrm{~s}$ (mean [SD], 242.6 [36.7] s). In the group in which the straight catheter was applied, more applications per vein were required (mean [SD], 1.49 [0.45] vs 1.25 [0.30]; $P<0.05)$. However, the total time of cryoballoon applications was similar in both groups (straight catheter, 1263.9 [394.8] s and crosier-shaped catheter, 1251.0 [307.8] s; TABLE2).

In the group in which the straight catheter was used, pacing from the distal electrode (poles 1-2) was favored in all 110 patients (100\%). In the other group, the electrode poles used for

TABLE 2 Comparison of the straight and crosier-shaped catheter techniques for phrenic nerve stimulation

\begin{tabular}{|c|c|c|c|c|}
\hline \multicolumn{2}{|l|}{ Parameter } & Straight catheter & Crosier-shaped catheter & $P$ value \\
\hline \multicolumn{2}{|l|}{ Percentage of acute PVI, mean (SD) } & $98.1(0.08)$ & $99.3(0.04)$ & 0.63 \\
\hline \multicolumn{2}{|c|}{ Total time of cryoballoon application, s, mean (SD) } & $1263.9(394.8)$ & $1251(307)$ & 0.73 \\
\hline \multicolumn{2}{|c|}{ Cryoballoon applications per vein, mean (SD) } & $1.49(0.45)$ & $1.25(0.30)$ & $<0.05$ \\
\hline \multicolumn{2}{|c|}{ Pacing threshold with 2-ms impulse, mA, mean (SD) } & $6.7(4.9)$ & $4.8(3.7)$ & $<0.01$ \\
\hline \multicolumn{2}{|c|}{ Total procedure time, min, mean (SD) } & $72.6(22.8)$ & $64.4(14.8)$ & $<0.01$ \\
\hline \multicolumn{2}{|c|}{ Min. temperature in $L S P V,{ }^{\circ} \mathrm{C}$, mean (SD) } & $-48.3(5.4)$ & $-49.1(8.1)$ & 0.39 \\
\hline \multicolumn{2}{|c|}{ Min. temperature in LIPV, ${ }^{\circ} \mathrm{C}$, mean (SD) } & $-45.1(7.5)$ & $-43.9(6.6)$ & 0.23 \\
\hline \multicolumn{2}{|c|}{ Min. temperature in $\mathrm{RSPV},{ }^{\circ} \mathrm{C}$, mean (SD) } & $-49.1(7.4)$ & $-48.7(6.7)$ & 0.67 \\
\hline \multicolumn{2}{|c|}{ Min. temperature in $\mathrm{RIPV},{ }^{\circ} \mathrm{C}$, mean (SD) } & $-45.6(14.1)$ & $-45.8(14.6)$ & 0.89 \\
\hline \multirow[t]{2}{*}{ Catheter positioning time, $\mathrm{n}(\%)$} & $<30 \mathrm{~s}$ & $94(85.5)$ & $88(81.5)$ & \multirow[t]{2}{*}{0.42} \\
\hline & $>30 \mathrm{~s}$ & $16(14.5)$ & $20(18.5)$ & \\
\hline \multicolumn{2}{|c|}{ Atrial capture during PNS (in sinus rhythm), n (\%) } & $10(11.5)$ & $24(29.6)$ & $<0.01$ \\
\hline \multicolumn{2}{|c|}{ Any correction maneuver during PNS, $\mathrm{n}(\%)$} & $35(31.8)$ & $21(19.4)$ & $<0.05$ \\
\hline \multicolumn{2}{|c|}{$\begin{array}{l}\text { Termination of cryoballoon application due to catheter dislocation, } \\
\mathrm{n}(\%)\end{array}$} & $12(10.9)$ & $6(5.5)$ & 0.16 \\
\hline \multicolumn{2}{|c|}{ Switch to another PNS technique, $n(\%)$} & $8(7.2)$ & $2(1.9)$ & 0.06 \\
\hline \multicolumn{2}{|l|}{ Phrenic nerve palsy, n (\%) } & $5(4.5)$ & $8(7.4)$ & 0.37 \\
\hline
\end{tabular}

Abbreviations: LIPV, left inferior pulmonary vein; LSPV, left superior pulmonary vein; Min., minimal; PNS, phrenic nerve stimulation; PVI, pulmonary vein isolation; RIPV, right inferior pulmonary vein; RSPV, right superior pulmonary vein 
FIGURE 3 Comparison of catheter positions in the right anterior oblique projection: A - straight, B - crosier-shaped
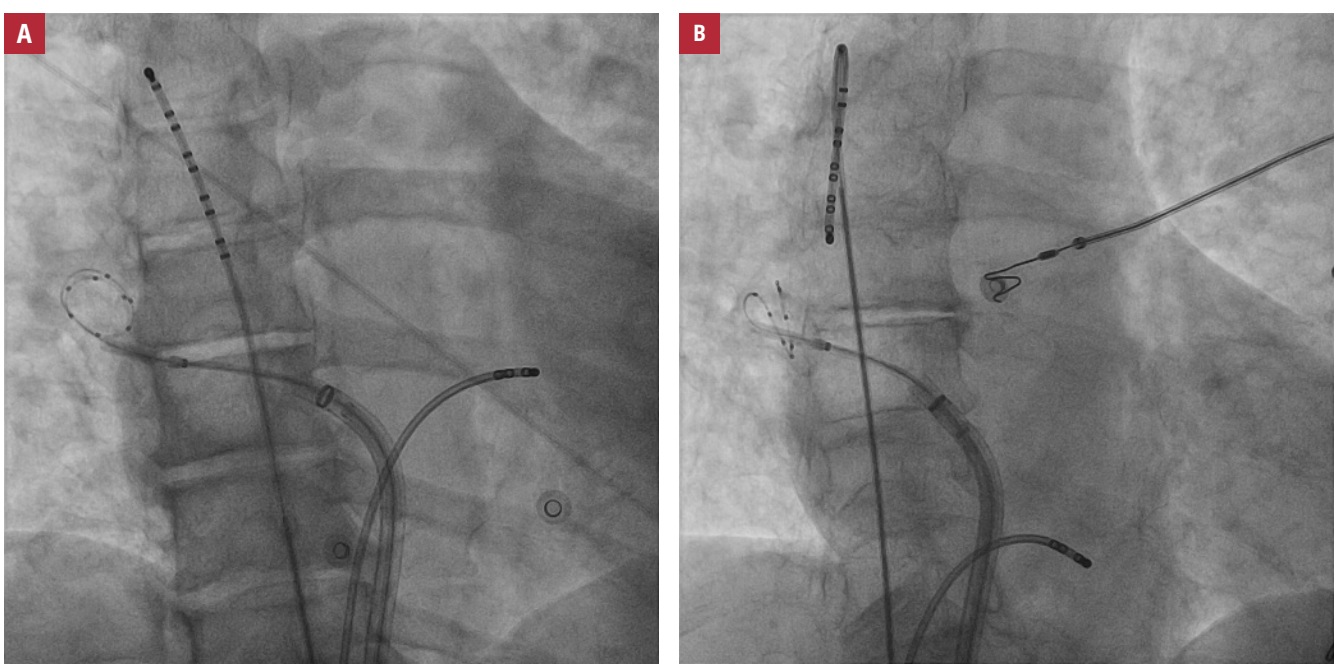

pacing varied among patients: 3 to 4 in $19 \mathrm{pa}$ tients (17.6\%), 5 to 6 in 64 (59.3\%), 7 to 8 in 22 (20.3\%), and 9 to 10 in 3 (2.8\%).

Neither the total time of cryoballoon applications nor the frequency of the phrenic nerve palsy was significantly affected by the position of the catheter during PNS. The positioning time was similar in both groups: it took less than 30 seconds in $85.5 \%$ of patients in whom the straight catheter was used and in $81.5 \%$ of patients in the other group. There was also no significant difference in the number of patients in whom the lack of phrenic nerve capture due to catheter dislocation resulted in the termination of cryoablation.

We found differences between study groups regarding the need to correct the catheter position. For the straight catheter, corrections were necessary in $31.8 \%$ of patients, while for the crosier-shaped catheter, only in $19.4 \%(P=0.036)$. Switching to a different technique due to recurrent catheter dislocations was required in $7.2 \%$ of patients in the straight-catheter group and in $1.9 \%$ of those in the crosier-shaped-catheter group $(P=0.06)$.

We also noted a difference between study groups in terms of the pacing threshold. It was lower in the crosier-shaped-catheter group than in the straight-catheter group (mean [SD], 4.8 [3.7] $\mathrm{mA}$ vs 6.7 [4.9] $\mathrm{mA} ; P=0.001)$. Additionally, the atrial capture was reached more often during PNS with the crosier-shaped catheter (29.6\% vs $11.5 \% ; P=0.004$ ).

Importantly, the groups showed different total procedure times. The procedures in which the crosier-shaped catheter was applied were shorter than those performed with the use of the straight catheter (mean [SD], 64 [14.8] min vs 72.6 [22.8] $\mathrm{min} ; P=0.006$ ). Interestingly, this parameter was similar in both groups if the analysis involved only patients in whom no correction of the catheter position was needed during PNS (66.0 [14.1] $\mathrm{min}$ vs 69.3 [25.1] $\mathrm{min} ; P=0.3$ ). The results are summarized in TABLE 2 .
DISCUSSION Our study showed that the straight and crosier-shaped catheter techniques for PNS are equal in terms of positioning time and effectiveness in the prevention of PNI. Also, they do not affect the time of cryoballoon application and the efficacy of PVI. This suggests that both techniques may be used interchangeably, according to operators' preference, and switching to the other technique is reasonable when the one chosen at first does not provide the adequate stability of pacing.

An important observation from our study is that the use of the crosier-shaped catheter is associated with the lower pacing threshold and more frequent atrial capture during pacing. As the pericardium separates the phrenic nerve from the superior vena cava at its junction with the right atrium, capturing the nerve in this region may require a higher amplitude than in the brachiocephalic vein and upper part of the superior vena cava where the nerve descends along their border covered with mediastinal pleura. ${ }^{14}$ The lower pacing threshold and more frequent atrial capture with the crosier-shaped catheter may be due to improved contact with the vein wall and the possibility to distend the vein by opening the curvature of the retroflexed catheter (FIGURE 3). Differences in pacing thresholds or atrial capture do not seem to affect patients' experience during PNS. However, patients may benefit more from one or the other technique depending on the anatomical variations in the course of the phrenic nerve.

Another significant difference between the 2 catheter techniques analyzed in our study was related to the need for switching to another technique due to catheter dislocations, instable phrenic nerve capture, or a high pacing threshold. More correction maneuvers and switching to another technique occurred in the group in which the straight catheter was used. This may be explained by the fact that the pressure applied to the vein by the retroflexed catheter with the opened curvature is probably 
higher. Moreover, the surface of the catheter that comes in contact with the vein wall is larger and, as a result, reduces friction forces and facilitates the smooth insertion of the catheter into the vein lumen. Therefore, the crosier-shaped catheter maintains its position better and is less likely to dislocate either spontaneously or, for example, due to a patient's cough or respiratory movements. This may be particularly important in procedures performed by a single operator, when the ability to correct the position of the catheter is limited during the first minutes of cryoablation. At that time, the operator may need to use both hands to apply constant pressure on a balloon catheter and a sheath to provide the adequate adhesion of a balloon to the vein ostium. The stable position of a catheter used for PNS allows the operator to focus on the proper cryoballoon application rather than on achieving stable phrenic nerve capture. Stable pacing could be achieved from more separated rather than adjacent electrode poles. However, no scientific evidence regarding such an approach has been available so far.

The total procedure time was significantly lower in the crosier-shaped-catheter group. $\mathrm{Pa}$ tients in whom the straight catheter was applied required significantly more cryoballoon applications per vein, which may be related to the increased need to stop cryoballoon applications prematurely due to loss of the phrenic nerve capture. However, the total time of application did not significantly differ between groups, so the differences in the total procedure time cannot be explained only by the necessity to apply additional freezes. Furthermore, no significant differences were found with regard to the time of catheter positioning. The shorter procedure time reported for the crosier-shaped catheter may be related to fewer corrections of the catheter position than in the case of the straight catheter. Such maneuvers as well as a 2-fold longer positioning time when switching to another technique prolong the procedure. Shortening the procedure time is particularly important for reducing the thromboembolic risk associated with catheter dwell time in the left atrium. These features of different catheter positions should be considered when pacing is used for the monitoring of phrenic nerve function.

Currently, there is no reliable method of predicting PNI prior to the procedure. ${ }^{11}$ However, Ichihara et $\mathrm{al}^{15}$ proved that preprocedural computed tomography and the evaluation of the distance between the ostium of the right superior pulmonary vein and the right pericardiophrenic bundle may help predict PNI. The second-generation cryoballoon significantly improved the success rate of ablation in patients with $\mathrm{AF}$ and reduced the procedure time, ${ }^{16}$ yet it tended to cause more PNIs and transient esophageal lesions. ${ }^{5}$ The higher risk of these complications requires operators to pay more attention to the effective prevention of PNI, and this is where the results of our research may prove particularly helpful.

Our study has several important limitations. First, it was an open-label study as the operator had to position a catheter manually. Second, the fact that all procedures were performed by the same electrophysiologist strongly limits the generalizability of our findings, but on the other hand, it helps avoid differences in technique and experience that would have to be considered if more operators were involved. Third, the study results were obtained with the use of 3 types of deflectable catheters and may be unreproducible with other types of steerable diagnostic catheters.

In conclusion, the straight and retroflexed, crosier-shaped catheters are equally effective in monitoring phrenic nerve function and avoiding its palsy. These 2 techniques do not differ significantly in terms of the time required for catheter positioning. The crosier-shaped catheter maintains its position better, and thus leads to fewer dislocations and reduces the number of correction maneuvers. As a result, its use is correlated with shorter procedure time. Moreover, the crosier-shaped catheter provides lower pacing thresholds. It also allows the operator to achieve atrial capture more frequently. Both techniques may be safely used for PNS during cryoballoon PVI in patients with $\mathrm{AF}$, and if the stable capture of the phrenic nerve cannot be maintained, switching to another technique seems to be reasonable.

\section{ARTICLE INFORMATION}

\section{CONFLICT OF INTEREST None declared.}

OPEN ACCESS This is an Open Access article distributed under the terms of the Creative Commons Attribution-NonCommercial-NoDerivatives 4.0 International License (CC BY-NC-ND 4.0), allowing third parties to download articles and share them with others, provided the original work is properly cited, not changed in any way, distributed under the same license, and used for noncommercial purposes only. For commercial use, please contact the journal office at kardiologiapolska@ptkardio.pl.

HOW TO CITE Kaczmarek K, Żuchowski B, Cygankiewicz I, et al. Straight and crosier-shaped catheter techniques for phrenic nerve stimulation during cryoballoon pulmonary vein isolation for the treatment of atrial fibrillation. Kardiol Pol. 2019; 77: 868-874. doi:10.33963/KP.14908

\section{REFERENCES}

1 Calkins H, Hindricks G, Cappato R, et al. 2017 HRS/EHRA/ECAS/APHRS/SOLAECE expert consensus statement on catheter and surgical ablation of atrial fibrillation: executive summary. Europace. 2018; 20: 157-208.

2 Kuck KH, Brugada J, Fürnkranz A, et al. Cryoballoon or radiofrequency ablation for paroxysmal atrial fibrillation. N Engl J Med. 2016; 374: 2235-2245.

3 Żuchowski B, Kaczmarek K, Szumowski L, et al. Interventional treatment of atrial fibrillation - contemporary methods and perspectives. Expert Rev Med Devices. $2014 ; 11:$ 595-603.

4 Chierchia GB, Di Giovanni G, Ciconte G, et al. Second-generation cryoballoon ablation for paroxysmal atrial fibrillation: 1-year follow-up. Europace. 2014; 16 : 639-644.

5 Straube F, Dorwarth U, Vogt J, et al. Differences of two cryoballoon generations: insights from the prospective multicentre, multinational FREEZE Cohort Substudy. Europace. 2014; 16: 1434-1442.

6 Andrade JG, Khairy P, Guerra PG, et al. Efficacy and safety of cryoballoon ablation for atrial fibrillation: a systematic review of published studies. Heart Rhythm. 2011; 8: 1444-1451. 
7 Franceschi F, Dubuc M, Guerra PG, Khairy P. Phrenic nerve monitoring with diaphragmatic electromyography during cryoballoon ablation for atrial fibrillation: the first human application. Heart Rhythm. 2011; 8: 1068-1071.

8 Mondésert B, Andrade JG, Khairy P, et al. Clinical experience with a novel electromyographic approach to preventing phrenic nerve injury during cryoballoon ablation in atrial fibrillation. Circ Arrhythm Electrophysiol. 2014; 7: 605-611.

9 Kowalski M, Ellenbogen KA, Koneru JN. Prevention of phrenic nerve injury during interventional electrophysiologic procedures. Heart Rhythm. 2014; 11: 1839-1844.

10 Su W, Kowal R, Kowalski M, et al. Best practice guide for cryoballoon ablation in atrial fibrillation: the compilation experience of more than 3000 procedures. Heart Rhythm. 2015; 12: 1658-1666.

11 Parikh V, Kowalski M. Comparison of phrenic nerve injury during atrial fibrillation ablation between different modalities, pathophysiology and management. J Atr Fibrillation. 2015; 8: 59-65.

12 Meissner A, Maagh P, Christoph A, et al. ECG-Guided surveillance technique in cryoballoon ablation for paroxysmal and persistent atrial fibrillation: a strategy to prevent from phrenic nerve palsy. Int J Med Sci. 2016; 13: 403-411.

13 Ghosh J, Singarayar S, Kabunga P, McGuire MA. Subclavian vein pacing and venous pressure waveform measurement for phrenic nerve monitoring during cryoballoon ablation of atrial fibrillation. Europace. 2015; 17: 884-890.

14 Mears JA, Lachman N, Christensen K, Asirvatham SJ. The phrenic nerve and atrial fibrillation ablation procedures. J Atr Fibrillation. 2009; 2: 19-36.

15 Ichihara N, Miyazaki S, Iwasawa J, et al. Prevalence and pre-procedural predictors associated with right phrenic nerve injury in electromyography-guided, second-generation cryoballoon ablation: single large balloon and single 3-minute freeze techniques. JACC Clin Electrophysiol. 2016; 2: 508-514.

16 Fürnkranz A, Bordignon S, Dugo D, et al. Improved 1-year clinical success rate of pulmonary vein isolation with the second-generation cryoballoon in patients with paroxysmal atrial fibrillation. J Cardiovasc Electrophysiol. 2014; 25: 840-844. 